\title{
Morphological study of the hoof in yak
}

\author{
X. Yang ${ }^{1}$, X. Ren ${ }^{2}$, S. Yu' ${ }^{2}$, Y. Cui ${ }^{1}$ \\ ${ }^{1}$ Laboratory of Animal Anatomy and Tissue Embryology, Department of Basic Veterinary Medicine, \\ Faculty of Veterinary Medicine, Gansu Agricultural University, Lanzhou, Gansu, China \\ ${ }^{2}$ Gansu Province Livestock Embryo Engineering Research Centre, Department of Clinical Veterinary Medicine, \\ Faculty of Veterinary Medicine, Gansu Agricultural University, Lanzhou, Gansu, China
}

[Received: 5 August 2015; Accepted: 15 September 2015]

\begin{abstract}
The fore- and hindlimb of yak have been studied by the gross anatomical methods and standard histological techniques. The artery of ungula was also determined by $X$-ray and acrylonitrile-butadiene-styrene casting. Anatomical features of the forelimb of yak resemble those of hindlimb, including hoof periphery, hoof coronal, hoof wall, hoof sole, and hoof sphere. The forelimb and hindlimb are almost the same in histological structure. The epidermis comprised all 5 strata: stratum corneum, stratum lucidum, stratum granulosum, stratum spinosum and stratum basale. The papillary layers and dermal lamellae contained a variable amount of capillary as well as collagen and elastic fibres. Many venules and arteriovenous anastomosis were distributed among the reticular layer. Subcutaneous tissue was composed of rich adipose and connective tissue in hoof periphery, hoof coronal, and, especially, hoof sphere. Major arteria in forelimb of yak include arteria digitalis palmaris communis III and arteriae digitales palmares propriae III and IV axialis. Those in hindlimb include arteria digitalis plantaris communis III and arteria digitalis plantaris propria III and IV axialis. Our findings highlight the main morphological features of yak and provide a morphological basis useful to researchers using yak hoof. (Folia Morphol 2016; 75, 3: 400-408)
\end{abstract}

Key words: forelimb, hindlimb, artery, anatomy, histology, yak

\section{INTRODUCTION}

The hoof is considered as the most important organ for supporting weight and for movement, and there are considerable variation in its shape, size, histology and arteries in different species of animals. In previous studies, a number of descriptions of the anatomy and histology of hoof exist for the different species.

In bovine, the properties of the suspensory and supporting structures of hoof are very important. An essential component is the shock-absorbing digital cushion situated under the distal phalanx [18]. A hairless area of epidermis is present on the posterior aspect of cattle hooves, which reveals three thick and well-demarcated cellular layers: 1) a glassy-clear stratum corneum; 2) the underlying epidermal cell layers; 3 ) the dermis [22].
The hooves in equine have been frequently mentioned $[6,10,13,15-17]$, the hoof wall consists of three layers: the stratum externum, stratum medium, and stratum internum. The stratum medium is the thickest of the three layers and is characterised by its tubular and intertubular horn structure. It is the main load support system of the equine foot and serves to transfer ground reaction forces to the bony skeleton [10].

In camel, the claw contains three parts: the fold, the wall and the sole. The fore digital pad is more developed than the hind one and both of them contain epidermis, dermis and subcutis $[4,5]$.

In elephant, the feet are equipped with large subcutaneous cushions. The cushions contain white and yellow adipose tissue and fibrous connective tissue. The micromorphology of elephant feet cushions 
Table 1. Specimens examined

\begin{tabular}{lcccc}
\hline Number & Sex & Age & Origin & Used for \\
\hline 7 & Male & $3-5$ years & Xining, Qinghai & Dissection \\
5 & Female & $5-9$ years & Linxia, Gansu & Histology \\
3 & Female & $4-6$ years & Gannan, Gansu & X-ray, ABS casting \\
\hline
\end{tabular}

ABS - acrylonitrile-butadiene-styrene

resembles that of digital cushions in cattle but not that of digital cushions in horse [25].

The vascular system of hoof in many kinds of species has been demonstrated, including bovine [23], equine [12, 16], camel [5, 24, 26], swine [11] and sheep [3].

In addition to providing meat, milk, and yakwool, yak also can be used for transporting goods and materials and for riding. It is the most important vehicle at the plateau area. However, no information is yet available on the morphological details of hoof and its arteries in yaks. Herein, to fill the gap we investigate the morphology of the hoof and its arteries in yaks and provide detailed morphological information of how the yaks adapt the plateau terrain.

\section{MATERIALS AND METHODS}

\section{Animals and dissections}

The forelimbs and hindlimbs of 15 yaks were examined after formalin fixation or deep freezing, using gross anatomical methods, standard histological techniques, X-ray, and acrylonitrile-butadiene-styrene (ABS) casting method (Table 1). Seven 3.5 years yaks that had lived in Xining, Qinghai were dissected and observed using stereoscopic microscope. Procedures involving animals were approved by the institutional animal care and use committee and complied with the Laboratory Animal Management Principles of China. All animals were euthanised by intravenous injection of pentobarbital sodium (150 mg/kg body weight).

\section{Sample preparation for histological examination}

Sections of fore- and hindlimbs of five 5.9 years old individuals from Linxia, Gansu were cut sagittally and tissues were excised and fixed in buffered formalin.

\section{Routine histology and specific staining methods}

Tissue samples from yaks were dehydrated, embedded in paraffin, sectioned at a thickness of $6 \mu \mathrm{m}$, deparaffinised, and stained with various staining methods. They were stained with haematoxylineosin (HE), Masson's trichrome, Weigert-Van Gieson
(W-VG) and Sacpic. Parts of epidermal lamellae were visualised by using polarised light microscope. Light microscopic observations and section images acquisition were performed using Olympus DP71 Light Microscope (including DP control and Image-Pro Express, Japan).

\section{X-ray and ABS casting}

To show the artery in the forelimb and hindlimb, this study used the injection-corrosion casting technique and angiography, separately. The artery orifices were cannulated, then four different concentrations of $A B S$ solutions ( $3 \%$ ABS in acetone, $5 \%$ ABS in acetone, $10 \%$ ABS in acetone and $15 \%$ ABS in acetone) and $20-25 \%$ BaSO4 were injected by hand under controlled pressure of $60-90 \mathrm{~mm} \mathrm{Hg}$, respectively. Then the ungulae that were injected with $A B S$ solution were placed in $30 \%$ hydrochloric acid to corrupt the soft tissue, and then flushed with a fine jet of tap water to remove the residue from the casts. After final cleaning and removal of the residue, photos of the casts were taken. Angiograms of the intact ungulae which were injected with 20-25\% BaSO4 were obtained using a mobile high frequency X-ray generator (Model KX-50vet, Shanghai, China) by taking roentgenograms in different directions. The distance was $70 \mathrm{~cm}$, exposure time $0.08-0.1 \mathrm{~s}$, being set at $40 \mathrm{kV}$, and at $32 \mathrm{~mA}$.

\section{RESULTS}

\section{Anatomical findings}

The hoof of yak consists of hoof capsule (epidermis), hoof corium (dermis and hypodermis) and phalanx (Fig. 1). Distal phalanx and about 1/2 middle phalanx are covered by hoof capsule (Fig. 2). Anatomical features of the forelimb of yak resemble those of hindlimb, including hoof periphery, hoof coronal, hoof wall, hoof sole, and hoof sphere (Figs. 1, 2). Parts of anatomic parameters of hoof in yak are shown in Table 2.

The soft, nearly white epidermis of hoof periphery is the border of glabrous skin and pileous skin. Numbers of funnel-shaped orifices of horn tubule regularly distribute in epidermal internal surface. The 


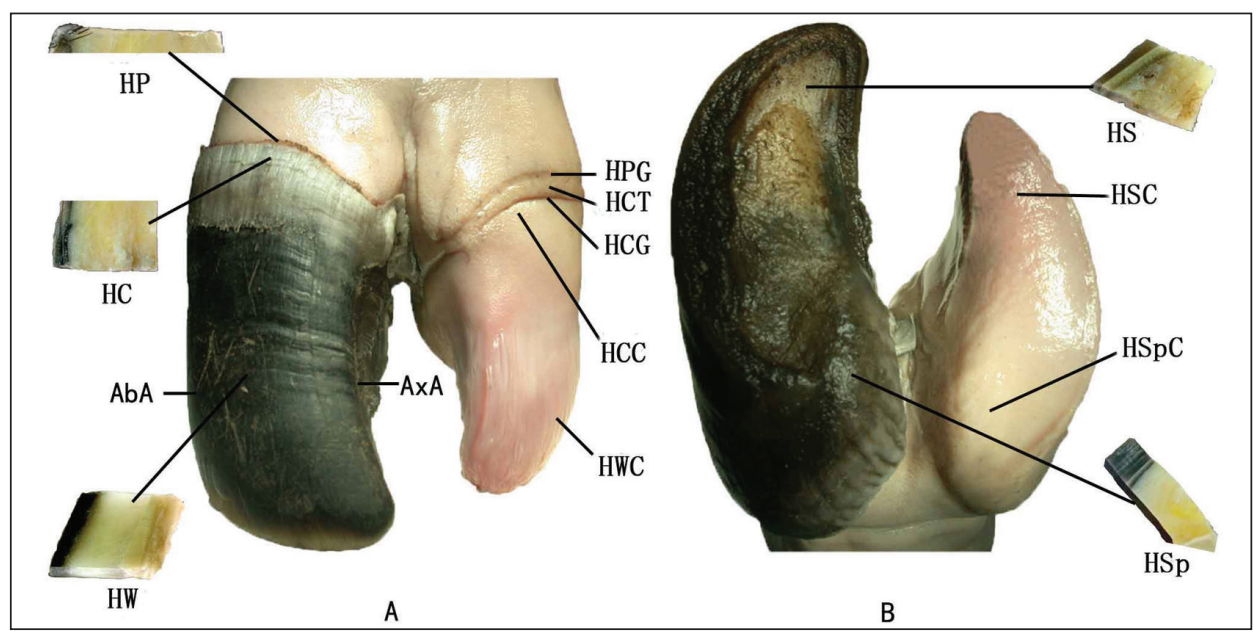

Figure 1. Anatomic structure of hoof in adult yak; A. The dorsal aspect; HP — hoof periphery; HC — hoof corona; HW — hoof wall; HPG — hoof periphery groove; HCT — hoof coronal tori; HCG — hoof coronal groove; HCC — hoof coronary corium; HWC — hoof wall corium; AbA — the abaxial aspect; AxA — the axial aspect; $\mathbf{B}$. The palmar aspect; HS — hoof sole; HSC — hoof sole corium; HSp — hoof sphere; HSpC — hoof sphere corium.

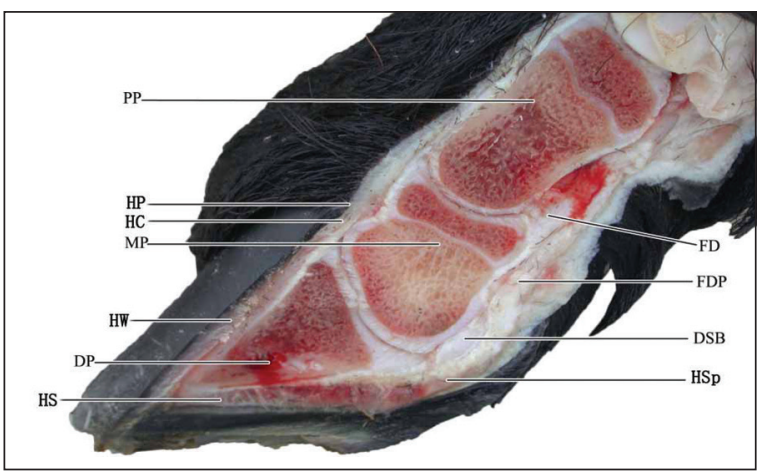

Figure 2. Longitudinal section of hoof in adult yak; DP — distal phalanx; MP — middle phalanx; PP — proximal phalanx; DSB — distal sesamoid bone; the other letter marks are the same as Figure 1.

semicircular, raised perioplic grooves are visible clearly in the perioplic corium (Fig. 1). Dermal papillae residing within the perioplic grooves are short and thinner, running to the medial of horn tubule.
The hoof coronal epidermis is bit soft, darker than those in hoof periphery, banded with different width. There are also a large number of orifices of horn tubule which resemble those of hoof periphery in coronary groove (Fig. 3). The coronary tori in coronary corium separate the hoof periphery and coronal, within it there are many dermal papillae.

The hoof wall epidermis is the hardest area of hoof capsule, including enamel layer, stratum coronarum, and stratum lamellatum (Fig. 3). A large number of horn tubule and intertubular horn are distributed in stratum coronarum. The density of horn tubule is decreasing gradually from outside to inside, and there lost horn tubule inside. There exist numbers of laminated horny laminae which are perpendicular to the hoof wall and distal phalanx in stratum lamellatum, but there are no secondary laminae (Fig. 4).

Table 2. Anatomic parameters of the hoof in yaks

\begin{tabular}{lc}
\hline Article & Anatomic parameters \\
\hline Circumferential length of hoof coronal epidermis [cm] & $15.69 \pm 0.82$ \\
Circumferential length of hoof wall epidermis [cm] & $14.85 \pm 0.72$ \\
Circumferential length of hoof sole epidermis [cm] & $16.27 \pm 0.90$ \\
Circumferential length of hoof coronal dermis [cm] & $11.58 \pm 0.73$ \\
Circumferential length of hoof wall dermis [cm] & $10.60 \pm 0.73$ \\
Circumferential length of hoof sole dermis [cm] & $15.38 \pm 0.74$ \\
Maximum length of lamella [cm] & $5.32 \pm 0.34$ \\
Minimum length of lamella [cm] & $1.37 \pm 0.42$ \\
Maximum height of lamella [cm] & $2.11 \pm 0.11$ \\
Minimum height of lamella [cm] & $0.24 \pm 0.06$ \\
Number of lamella & $762.63 \pm 47.06$ \\
\hline
\end{tabular}




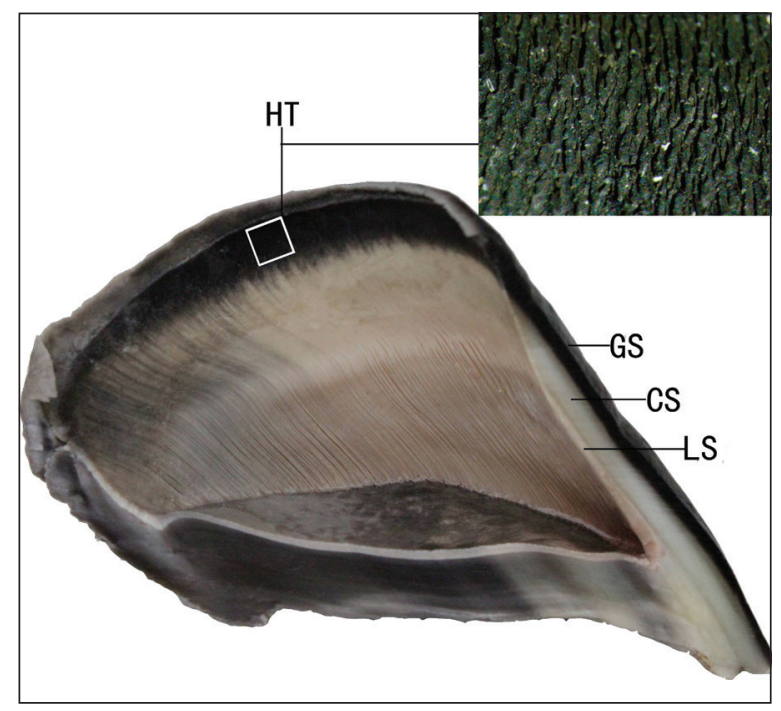

Figure 3. Longitudinal section of hoof epidermis in adult yak; HT — horn tubule; GS — glaze stratum; SC — coronary stratum; LS - lamellar stratum.

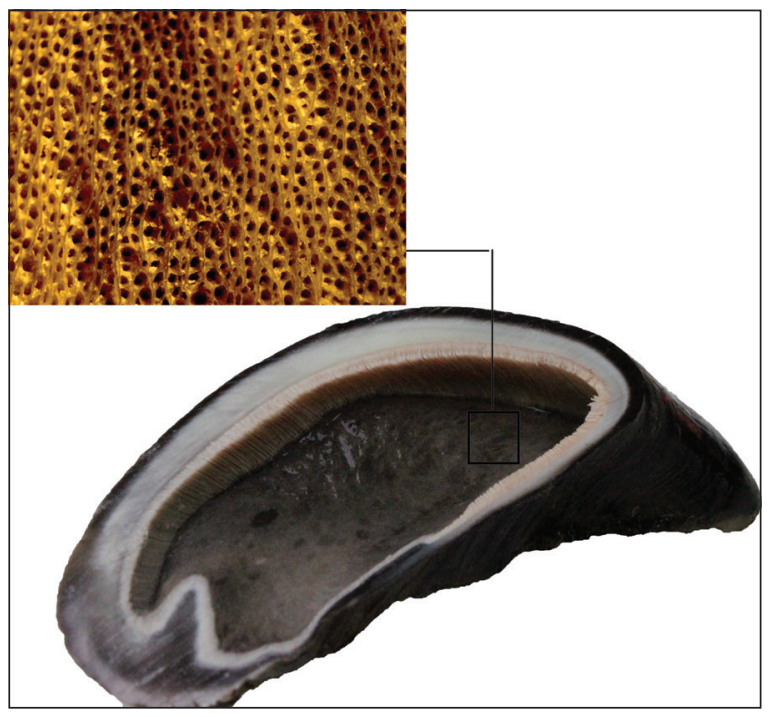

Figure 5. Transverse section of hoof epidermis in adult yak. Inner pane structure shows the plantar horn tubule.

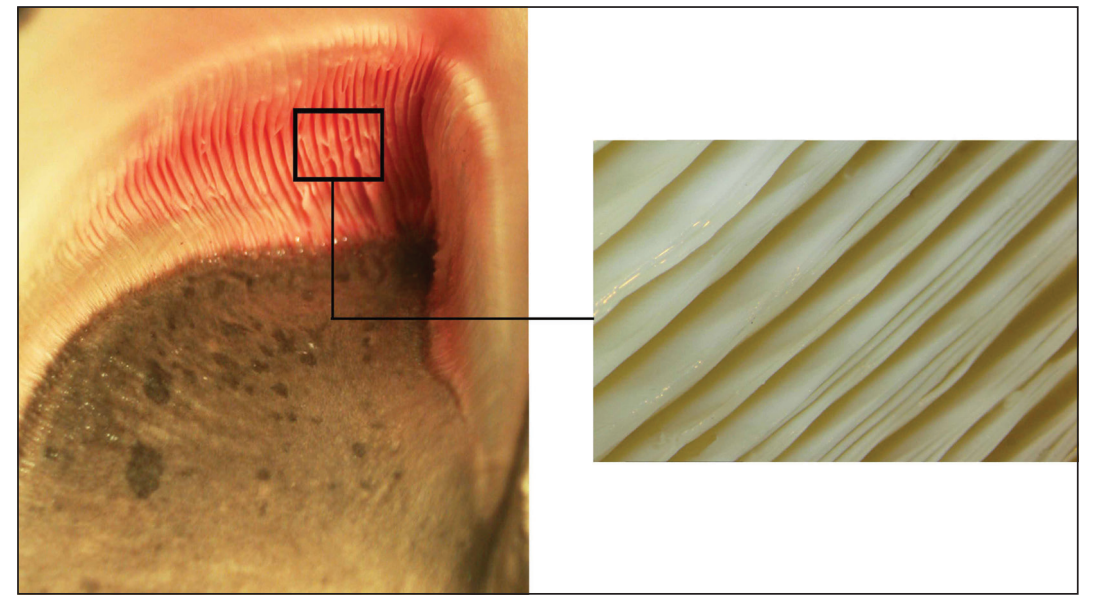

Figure 4. Lamellae of inner hoof wall in adult yak.
The back of hoof sole connects with hoof sphere, and the white line at lateral side separates the hoof sole and wall. The orifices of horn tubule and the dermal papillae are directed towards to the hoof toe (Fig. 5).

The hoof sphere is raised torus-sharped, locate in the back of the hoof. There are lots of irregular orifices of horn tubule (Fig. 6). The dermal papillae are short and irregular.

\section{Histological findings}

The forelimb and hindlimb are almost the same in histological structure. The epidermis comprises all 5 strata: stratum corneum, stratum lucidum, stratum granulosum, stratum spinosum and stratum basale (Fig. 7A, C, G, M, O). The dermis contains the papillary and reticular layers.

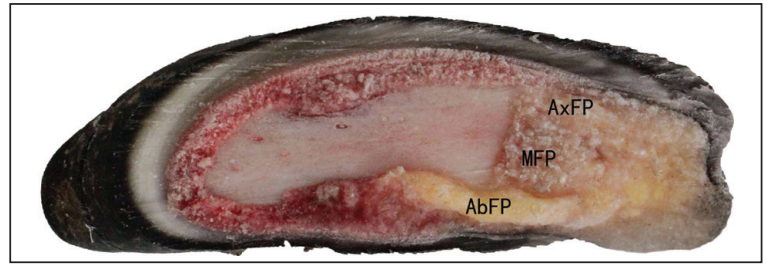

Figure 6. Transverse section of hoof in adult yak; AbFP — abaxial fat pads; MFP — middle fat pads; AxFP — axial fat pads.

\section{The epidermis}

The horny layer is made of elliptical anucleated cells, arranged scaly in the hoof periphery (Fig. 7B). The horny layer of hoof coronal contains two types of cells, one is slightly keratinised, the other is strongly keratinised (Fig. 7D). The stratum corneum is subdivided in two zones, 


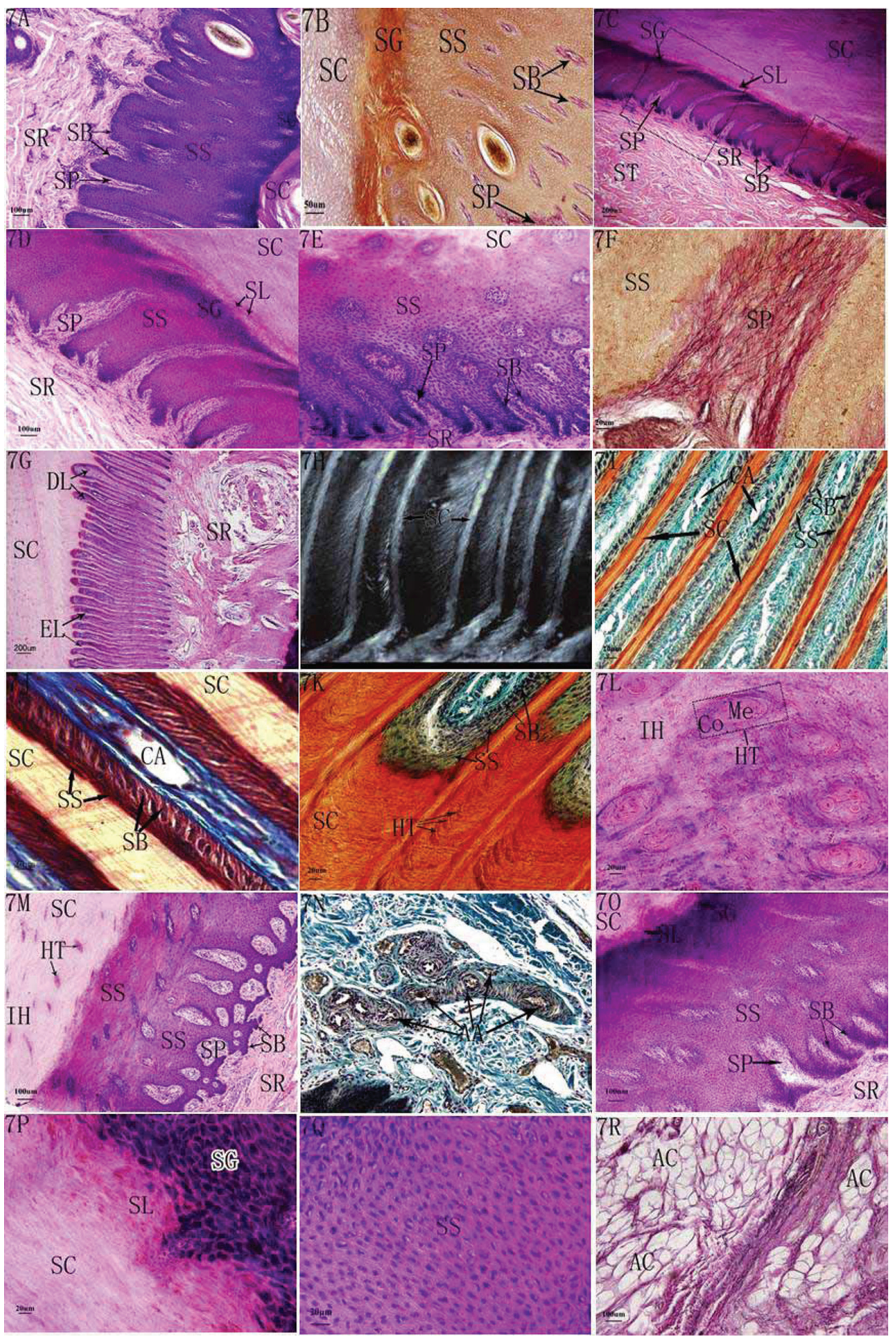

Figure 7. Histological section of hoof in adult yak; A. The histological section of hoof periphery $(H E, 100 \times)$; B. The histological section of hoof periphery epidermis (WVG, 200×); C. The histological section of hoof corona $(H E, 40 \times)$; D. Enlarge view of corresponding position in the big pane of panel $\mathrm{C}(\mathrm{HE}, 100 \times)$; E. Enlarge view of corresponding position in the small pane of panel C $(\mathrm{HE}, 200 \times)$; $F$. Stratum papillare and stratum spinosum of hoof corona (WVG, $400 \times)$; $\mathbf{G}$. The histological section of hoof wall $(\mathrm{HE}, 40 \times)$; $\mathbf{H}, \mathbf{I}, \mathbf{J}, \mathbf{K}$. The histological section of lamellar stratum of hoof wall(micropolariscope, Sacpic, Masson's trichrome stain and Sacpic, $400 \times$ ); L. The histological section of coronary stratum of hoof wall $(\mathrm{HE}, 400 \times)$; M. The histological section of hoof sole $(\mathrm{HE}, 100 \times)$; N. Arteriovenous anastomosis of hoof sole corium (Sacpic, $200 \times)$; 0. The histological section of hoof sphere (HE, 100 $)$ ); P. Stratum corneum, stratum lucidum and stratum granulosum of hoof sphere epidermis(HE, $400 \times)$; $\mathbf{0}$. Stratum spinosum of hoof sphere epidermis $(H E, 400 \times)$; $\mathbf{R}$. Subcutaneous tissue of hoof sphere $(W V G, 100 \times)$; SC — the stratum corneum; SL — the stratum lucidum; SG — the stratum granulosum; SS — the stratum spinosum; SB — the stratum basale; $\mathrm{SP}$ — the stratum papillare; SR — the stratum reticulare; ST — the subcutaneous tissue; HT — the horn tubule; IH — the intertubular horn; Co — the cortex; Me — medulla; AA — the arteriovenous anastomosis; CA — the blood capillary; DL — the dermal lamellae; EL — the epidermal lamellae; $\mathrm{AC}$ - adipose cell. 
the horn tubule, which consist of cortex and medulla, and the intertubular horn in hoof wall, hoof sole, and hoof sphere (Fig. 7H, I, J, K, M, P). The hoof wall epidermis only contains stratum corneum and stratum lamellatum. Polarisation light microscopy reveals that in the epidermal lamellae horny layer is the centre and flanked by the spinous layer and basal layer (Fig. 7H). The stratum lucidum is a pink uniform band, only present in hoof coronal and hoof sphere (Fig. 7D, P), using HE staining. The stratum granulosum exists in hoof periphery, hoof coronal, and hoof sphere (Fig. 7B, F, P). On HE-stained sections, the granular layer is conspicuous, consisting of numbers of fusiform dark-blue cells (Fig. 7P). The cells in the spinous layer become polygonal, with a round or elliptical nucleus (Fig. 7B, E, F, K, Q). The basal cell layer is made of cuboidal and columnar cells, which are arranged regularly. The spinous layer in the hoof wall consists of 2-3 layers of cells in the middle of the epidermal lamellae, whereas more layers of cells can be found in the summit and the base of the lamellae (Fig. 7H, I, J, K). The columnar basal cells are lying perpendicular to spinose cells (Fig. 7l, J), using Masson's and Sacpic staining. The dermal lamellae and epidermal lamellae fit together well to form a tight junction.

\section{The dermis}

The dermis is a connective tissue subdivided in two zones, the papillary dermis and the reticular dermis. The dermis contains numerous capillary, elastic fibres and collagen fibres. Additionally, there are arteriovenous anastomoses in the reticular dermis. The hypodermis contains a variable amount of fat in the hoof sphere (Fig. 7F, N, R).

\section{Arteries in fore- and hindlimb}

\section{The arteries in the forelimb}

The main arteries in the forelimb of yak include palmar common digital artery III and abaxial palmar digital artery III and IV (Fig. 8).

Palmar common digital artery III is the continuation of arteria mediana, and forms the two branches, digital arteries III and IV (Fig. 8), in the gap, upon the interphalangeal joint, below the proximal phalanx. Then palmar common digital artery III divides into arteriae palmares propriae III and IV axial (Fig. 8), which supply the muscles, fat, and skin in the pars axialis.

Digital arteries III and IV form vasoganglion in the hoof sphere, and combine with abaxial palmar digital arteries III and IV and the branches of arteriae

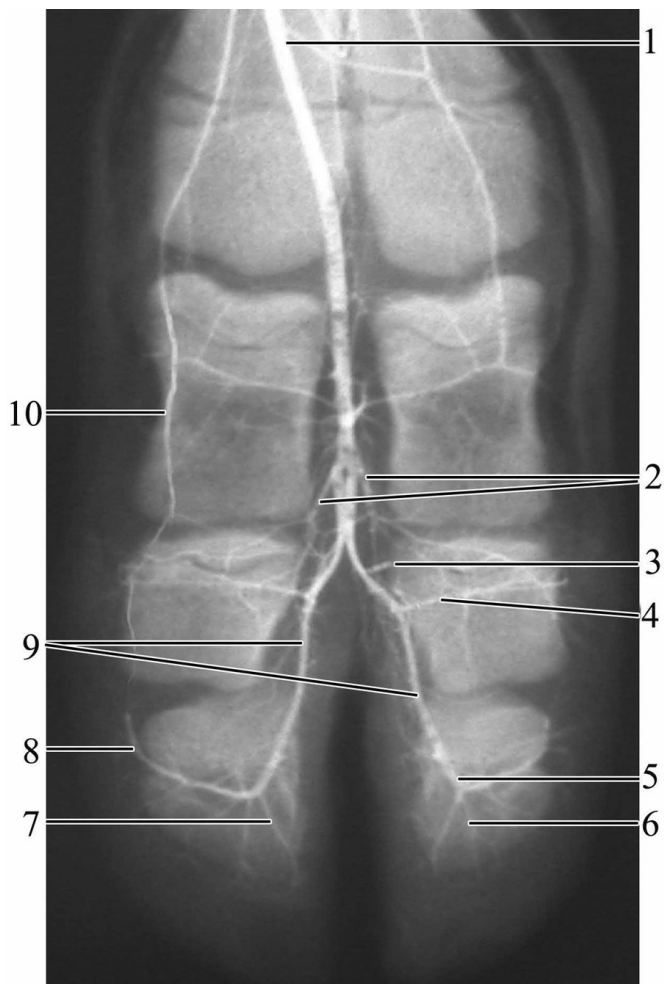

Figure 8. Distribution of arterial vessel in forelimb of yak (Dorsalis); 1 - palmar common digital a. III; 2 - arteria digital III and IV digit; 3 - artery palmaris middle phalanx; 4 - artery dorsi-middle phalanx; 5 - turning angle of artery replication propriae III digit; 6 - artery ungula point side ramus; 7 - artery ungula point ramus; 8 - artery replication propriae IV digit; 9 - axial palmar digital a. III and IV; 10 - abaxial palmar digital a. IV.

palmares propriae III and IV axial to form a network in the connective tissue of hoof sphere.

Arteriae palmares propriae III and IV axial fork artery palmaris-middle phalanx and artery dorsi-middle phalanx at the proximal middle phalanx, and divide into arteries replication propriae III and IV.

The branches of arteria palmaris-middle phalanx combine with the branches of arteriae digitalis III and IV, supply the muscles and fat in the palmaris. The branches of artery dorsi-middle phalanx combine with the branches of abaxial palmar digital arteries III and IV, digital arteries III and IV, and arteriae replication propriae III and IV, supply dorsal muscle.

Arteriae palmares propriae III and IV axial traverse the orifice at the distal phalanx axis, form a sharp angle in the distal phalanx and arteriae replication propriae III and IV. Arteries replication propriae III and IV fork artery ungula point ramus and artery ungula point side ramus at the sharp angle towards hoof-head. The two arteries run along hoof-head and anastomose with each other to form 


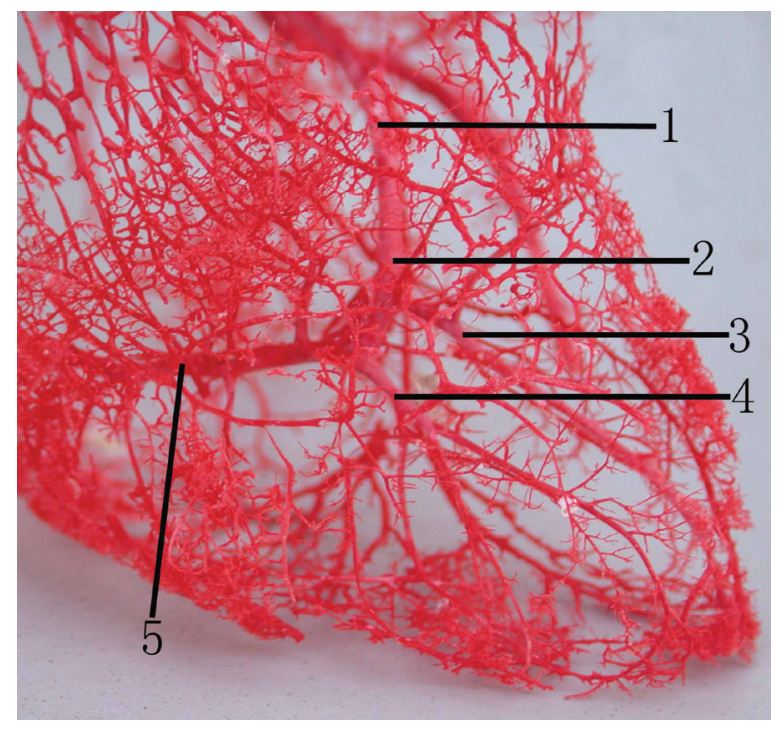

Figure 9. Distribution of arterial vessel in forelimb hoof point of yak (Dorsalis); 1 - axial palmar digital a. IV axial; 2 - turning angle of artery replication propriae IV digit; 3 - artery ungula point ramus; 4 - artery ungula point side ramus; 5 - artery replication propriae IV digit.

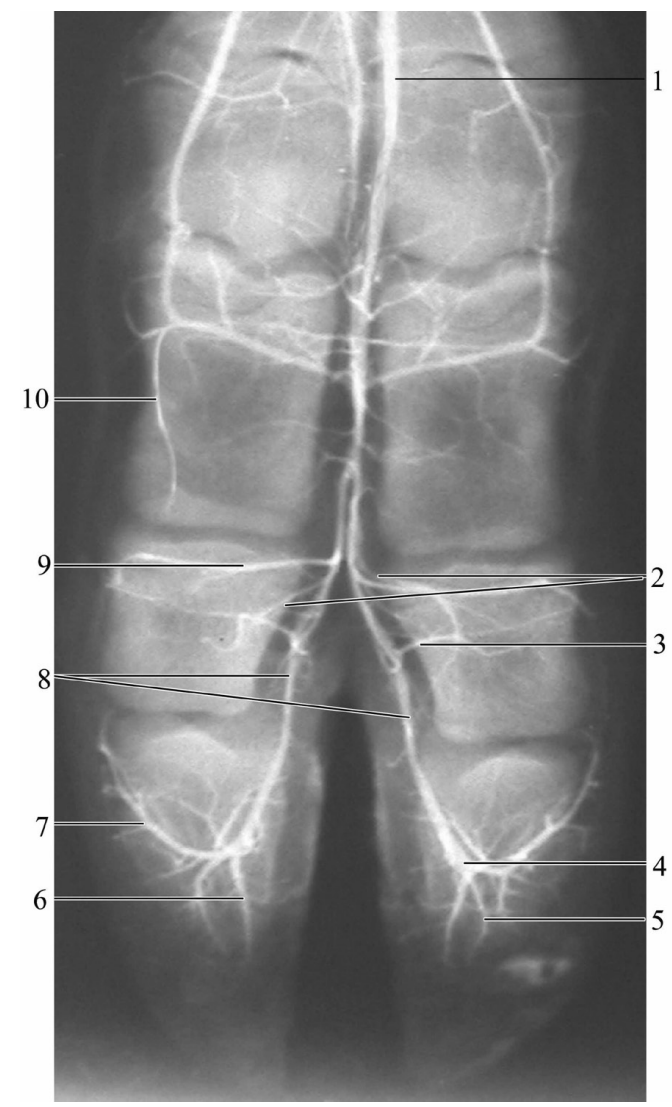

Figure 10. Distribution of arterial vessel in yak's hind limb (Dorsalis); 1 - artery digitalis dorso-pedis communis III; 2 - arteria digital III and IV digit; 3 - artery planta middle phalanx; 4 - turning angle; 5 - artery ungula point side ramus; 6 - artery ungula point ramus; 7 - artery replication propriae III digit; 8 - artery propriae III and IV pedis dorso-axial; 9 - artery dorsi-middle phalanx; 10 artery propriae III pedis far axial. cambered anastomosis (Fig. 9). The branches of arteries replication propriae III and IV and artery ungula point ramus form abundant vasoganglion in the pars axialis and pars abaxialis.

Abaxial palmar digital arteries III and IV run in the pars abaxialis of the hoof, combine with the branches of arteriae replication propriae III and IV, supply muscles at the pars abaxialis.

\section{The artery in the hindlimb}

The main arteries in the hindlimb include arteriae digitalis dorso-pedis communis III and arteriae propriae III and IV pedis far axial (Fig. 10). Arteriae digitalis dorsopedis communis III originates from the trunk of anterior tibial artery. Arteriae digitales III and IV, artery dorsimiddle phalanx and artery planta middle phalanx are the branches of arteries propriae III and IV pedis dorso-axial (Fig. 10); the remainder are the same as the forelimb.

Blood vessels upon the 2/3 of hoof wall and in the hoof sole are dense; however, the vessels' density is obviously decreased in the lower $1 / 3$ of hoof wall (Figs. 11, 12).

\section{DISCUSSION}

In yak, the tubule density of the stratum coronarum of hoof wall is decreased gradually from outside to inside. This finding resembles that in horse [19]. In equine, the tubules of the stratum medium are arranged in 4 distinct zones based on the density of tubules in the intertubular horn [20]. Tubule density of the stratum medium has been defined as the number of tubules/unit area [20] and many researches have suggested that this parameter influences the macroscopic mechanical properties of hoof horn. For example, Geyer and Tagwerker [8] suggested that 'hardness' was related to tubule density in cattle and pig hoof.

Since the force of impact with the ground is transmitted proximally up the wall [21], the tubule density gradient across the wall appears to be a mechanism for smooth energy transfer, from the rigid (high tubule density) outer wall to the more plastic (low tubule density) inner wall, and ultimately to the distal phalanx. The observations in equine hoof of Kasapi and Gosline [10] suggest that tubules may facilitate the dehydration of the hoof wall at the distal surface, since dehydration rate was highest through the vertical surface [10]. The gradient of tubule density corresponds to the gradient of water content across the hoof wall [14]. These factors represent an optimum design for equine hoof wall as well as yak hoof wall. 


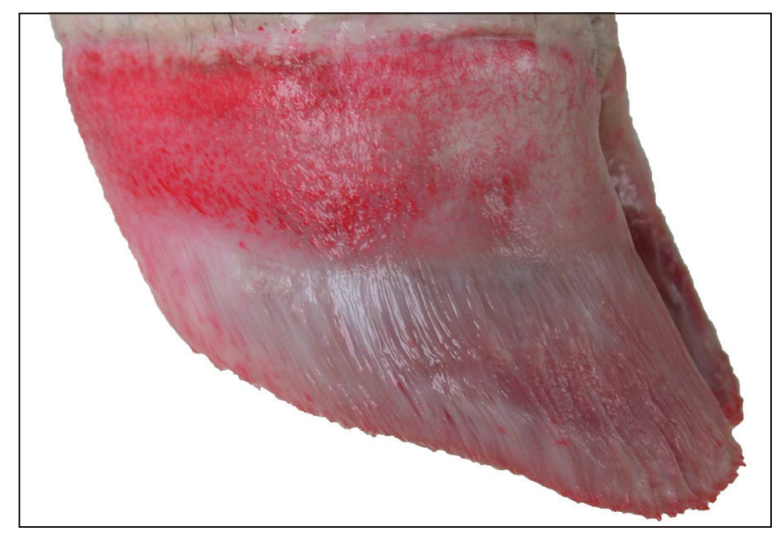

Figure 11. Distribution of arterial vessels in hoof wall corium of yak.

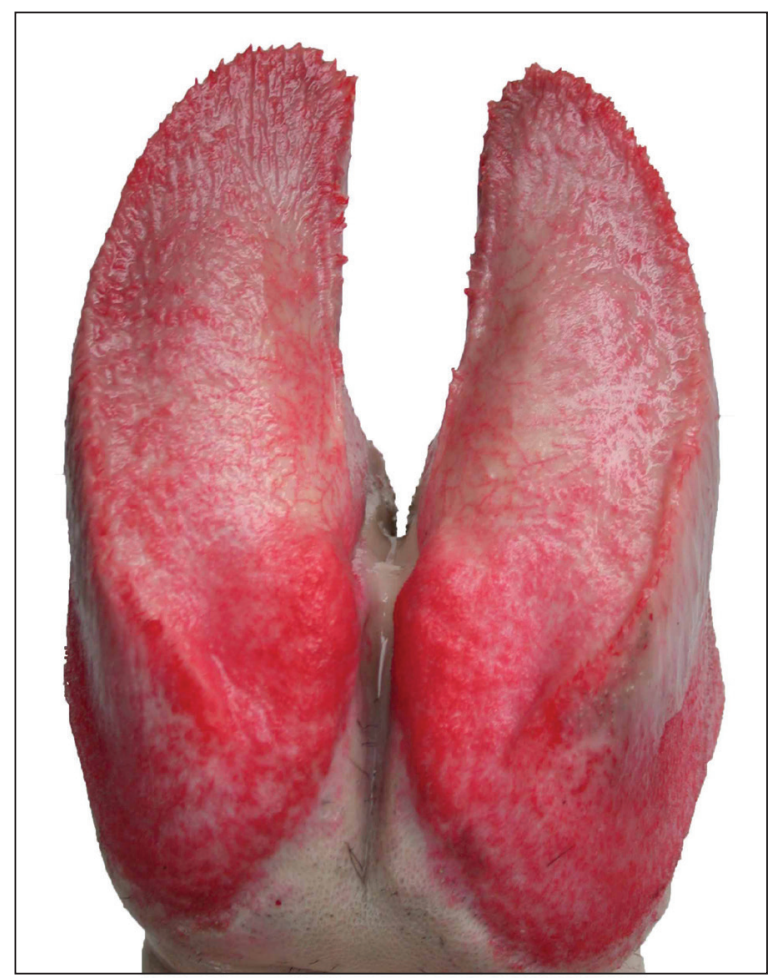

Figure 12. Distribution of arterial vessels in hoof sole corium of yak.

We measured the number of the lamellae of hoof wall in yak by the gross anatomical methods. Our observations that there is no secondary lamellae resemble those in cattle and swine [7], but not those in equine [16], elephant [25] and Bactrian camel [5]. The number of primary lamellae of hoof in yak, which is 762,63 , is far more than those in equine [16]. There are secondary lamellae in horses in the innermost layer of the hoof wall, after 550 to 600 primary epidermal lamellae, which project from its surface in parallel rows. This large surface area for the great secondary lamellae architecture helps reduce stress and ensures even energy transfer during peak loading of the equine foot [1].

In equine, large part of the body weight is carried by the suspensory apparatus attached to the hoof walls, only a small load rests on the sole and heel segment $[2,9]$.

The suspensory apparatus is less well developed in cattle, thus, the cushions must support a considerably greater proportion of the body weight. Digital cushions in cattle comprise resilient loose connective tissue with varying amounts of associated soft fat enclosed in an envelope of collagenous connective tissue [18].

In yak, there is some amount of adipose tissue in the hoof sphere. This structure, which as a dashpot, serves to absorb mechanical shock, store and return elastic strain energy, protect against local stress and keep pressures low, and reduces the damage to distal phalanx.

The observations of Daradka and Pollitt [6], who stated that the terminal part of the digital artery enters the solar canal of the distal phalanx via the paired sole foramina and unites with the artery of the opposite side to form the terminal arch deep within the bone, could not be supported by our findings, which showed that there was turning angle instead of terminal arch in yak hoof. Our findings resemble that in cattle [27]. We find that there are no difference in terms of the distribution of arterial vessels among bovids of different ages and breeds. But the distribution of arterial vessels in yak hoof is opposite to this in sheep [3], which suggests that the difference among different kinds of animals may be related to their environment.

\section{CONCLUSIONS}

In yak, palmar common digital artery III forms two branches: first, arteria digital III and IV digit, and subsequently arteria palmares propriae III and IV axial, in the fore limb. This structure may be related to the fact that forelimbs support more body weight than hindlimbs. Arteria digital III and IV digit can reach the hoof sphere directly and reduce the loss of energy. In the hindlimbs we find the same regulation.

Normal function of the bovine claw can be disrupted by changes in blood flow. The vascular involvement in claw lesions has been recognised. The vessels in the hoof can provide nutrients quickly and run blood circulation at the end of the sole. Where the more nutrients needed, the more vessels are 
present, which suggests that this structure will need to be protected more carefully.

\section{Acknowledgements}

This research was supported by the National Natural Science Foundation of China (31360594). We greatly appreciate the assistance of Professor Cui Yan (Laboratory of Animal Anatomy and Tissue Embryology, Department of Basic Veterinary Medicine, Faculty of Veterinary Medicine, Gansu Agricultural University, Lanzhou, Gansu, China) and the cooperation provided by Professor Yu Sijiu from the Gansu Province Livestock Embryo Engineering Research Centre (Gansu Agricultural University, Lanzhou, China). We would like to thank Duan Deyong (Lanzhou, China) for strong technical support, He Junfeng (Gansu Agricultural University, China) for kindly assisted in arranging the X-ray scans.

\section{REFERENCES}

1. Bertram JE, Gosline JM (1987) Functional design of horse hoof keratin: the modulation of mechanical properties through hydration effects. J Exp Biol, 130: 121-136.

2. Bruhnke J (1928) Vergleichende studie der statitsch beanspruchten lederhautteile des pferdehufes und der rinderklaue. Arch Wiss Prakt Tierheilk, 57: 324-334.

3. Chen R, Li T, Yang Y (2009) Arterial vessel and architecture of vascular bed in adult sheep hoof. Chinese J Anat, 32: 523-529.

4. Cui Y, Xie Z (1996) The anatomical structure of the digital pad of two-humped camel (Bactrianus camelus). Chinese J Vet Science, 16: 369-372.

5. Cuiyan (1996) The anatomic and histological structures of the claw in Bactrian camel (Camelus bactriamus). J Gansu Agricultural University, 31: 1.

6. Daradka M, Pollitt CC (2004) Epidermal cell proliferation in the equine hoof wall. Equine Vet J, 36: 236-241.

7. Fan ZF, Yu LH (1984) Morphology and structure of the digital cushion and ungulae in pigs. J Northeast Agricultural Coll, 41.

8. Geyer H, Tagwerker F (1986) The pig's hoof: its structure and alterations. Hoffmann-La Roche and Co., Bade, Switzerland.

9. Habermehl KH (1996) Haut and hautorgane. In: Nickel R, Schummer A, Seiferle E eds. Lehrbuch der Anatomie der Haustiere. Vol. 3. Paul Parey, Hamburg, pp. 485-492.
10. Kasapi MA, Gosline JM (1998) Exploring the possible functions of equine hoof wall tubules. Equine Vet J, 26: $10-14$.

11. Liu J (1982) Investigation into the origin of the dorsal metacarpal arteries of swine. Acta Vet Zootec Sin, 13: 141.

12. Nasu T, Yamanaka T, Nakai M, Ogawa H (1998) Scanning electron microscopic study of the vascular supply of the equine hoof. J Vet Med Sci, 60: 855-858.

13. Pollitt CC (1994) The basement membrane at the equine hoof dermal epidermal junction. Equine Vet J, 26: 399-407.

14. Pollitt CC (1995) Color Atlas of the Horse's Foot, MosbyWolfe, London.

15. Pollitt CC (1998) The anatomy and physiology of the hoof wall. Equine Vet Educ, 10: 318-325.

16. Pollitt CC (2004) Anatomy and physiology of the inner hoof wall. Clin Tech Equine Pract, 3: 3-21.

17. Pollitt CC (2010) The anatomy and physiology of the suspensory apparatus of the distal phalanx. Vet Clin North Am Equine Pract, 26: 29-49.

18. Räber M, Lischer CJ, Geyer H, Ossent P (2004) The bovine digital cushion: a descriptive anatomical study. Vet J, 167: 258-264.

19. Reilly JD, Collins SN, Cope BC, Hopegood L, Latham RJ (1998) Tubule density of the stratum medium of horse hoof. Equine Vet J, 26: 4-9.

20. Reilly JD, Cottrell DF, Martin RJ, Cuddeford D (1996) Tubule density in equine hoof horn. Biomimetics, 4: 23-26.

21. Thomason JJ, Biewener AA, Bertram JE (1992) Surface strain on the equine hoof wall in vivo: implications for the material design and functional morphology of the wall. J Exp Biol, 166: 145-168.

22. Ugel AR, Idler W (1970) Stratum granulosum: dissection from cattle hoof epidermis. J Invest Dermatol, 55: 350-353.

23. Vermunt JJ, Leach DH (1992) A scanning electron microscopic study of the vascular system of the bovine hind limb claw. N Z Vet J, 40: 146-154.

24. Wang J, Li T, Yan H, Fu D, Li H, Xie Z (2000) Anatomy of the deep veins of the forelimb in the Bactrian camel (Camelus bactrianus). J Lanzhou University (Natural Sciences), 36: 133.

25. Weissengruber GE, Egger GF, Hutchinson JR, Groenewald HB, Elsässer L, Famini D, Forstenpointner G (2006) The structure of the cushions in the feet of African elephants (Loxodonta africana). J Anat, 209: 781-792.

26. Yu H, Cui Y (1995) A study on vasculoarchitecture of digital region in two-humped camel (Camelus Bactrianus). Acta Zool Sin, 41: 256.

27. Yuan FL, Chao XY (2000) Studies on the pattern of cattle hoof vessels. J Ningxia Agricultural Coll, 21: 54. 